\title{
New Technology of Thermoplastic Coating for Osmotic Pump Tablets: Study on in vivo Drug Release
}

\author{
Chun Ping Yuan ${ }^{1,2}$ \\ ${ }^{1}$ National Pharmaceutical Engineering Research Center, China State \\ Institute of Pharmaceutical Industry, Shanghai, People's Republic of \\ China \\ 2 Research and Development Center, Sinopharm Group Guangdong \\ Medi-world Pharmaceutical Company Limited, Foshan, People's \\ Republic of China
}

Pharmaceut Fronts 2020;2:e1-e10.

\author{
Qing Hua Ge ${ }^{1}$ Ding Zhong Song ${ }^{1}$ Jian Qiang $X^{1}$
}

\author{
Address for correspondence Huimin Hou, PhD, National \\ Pharmaceutical Engineering Research Center, China State Institute of \\ Pharmaceutical Industry, 1111 Halley Road, Shanghai 201203, \\ People's Republic of China (e-mail: houhm@nperc.com).
}

\begin{abstract}
Keywords

- thermoplastic coating

- spray coating

- metformin hydrochloride

- nifedipine

- osmotic pump tablet

- pharmacokinetics

- in vivo-in vitro correlation

Aim The in vivo pharmacokinetics of thermoplastic-coated tablets prepared by a new technology of thermoplastic coating in Beagle dogs were studied, and the correlation between in vitro release and in vivo absorption was analyzed.

Methods The in vitro release profiles of metformin hydrochloride thermoplasticcoated tablets and nifedipine thermoplastic-coated tablets were investigated. The single-dose pharmacokinetic study of these tablets in Beagle dogs was performed, and the obtained results were separately compared with the data of conventional osmotic pump tablets reported in the literature.

Results Metformin hydrochloride thermoplastic-coated tablets and nifedipine thermoplastic-coated tablets displayed controlled drug-release characteristics and had a good in vivo-in vitro correlation in Beagle dogs, respectively. The literature-compared results further demonstrated that both thermoplastic-coated tablets had release characteristics of osmotic pump tablets in vivo.

Conclusion The thermoplastic-coated tablets could control drug release in vivo and it was further confirmed that the new thermoplastic coating technology could replace the spray coating of osmotic pump controlled-release tablets. This study provides a theoretical basis and practical support for the industrialization and clinical application of the new thermoplastic coating technology.
\end{abstract}

\section{Introduction}

The preparation process of a new thermoplastic coating technology (HHL method), ${ }^{1}$ the properties of the thermoplastic coating membrane, ${ }^{2}$ and the in vitro release profiles of thermoplastic-coated tablets ${ }^{3}$ have been published separately previously. In vitro release studies showed that thermoplastic-coated tablets possessed the same release mechanism (osmotic pressure drove the drug release) and the same release characteristics (zero-order release, unaf- fected by the release medium and stirring speed) and the same release rule (the release rate was inversely proportional to the membrane thickness but proportional to the porogen content) as the osmotic pump tablets (OPTs) prepared by the conventional spray coating, therefore displayed the same characteristics as OPTs. In addition, the zero-order drug release could be achieved by adjusting the membrane thickness and the porogen content. The above results preliminarily indicated that the thermoplastic coating technology might replace the conventional spray coating. received

November 9, 2019

accepted

December 6, 2019
DOI https://doi.org/

1055/s-0040-1701436.

ISSN 2628-5088.
(C) 2020 Georg Thieme Verlag KG Stuttgart . New York
License terms

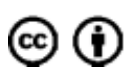


Metformin hydrochloride, a water-soluble drug, has been prepared as single-chamber OPTs while nifedipine, a waterinsoluble drug, is available as double-chamber OPTs on the market. We selected these two representative drugs to evaluate the applicability of this new thermoplastic coating technology. In this article, to verify the in vivo drug-release properties of thermoplastic-coated tablets, metformin hydrochloride thermoplastic-coated tablets (HHL-METF) and nifedipine thermoplastic-coated tablets (HHL-NIF) were prepared and their pharmacokinetics in Beagle dogs were investigated and compared with OPTs prepared by the conventional spray coating. The in vitro-in vivo correlation was also investigated to further confirm the substitutability of the HHL method, which laid a solid foundation for the industrialization and clinical application of the HHL method.

\section{Materials and Methods}

\section{Materials}

The standard substances of METF and NIF were purchased from the National Institute for Food and Drug Control (Beijing, China). NIF-d6 was purchased from Toronto Research Chemicals Inc. (Toronto, Canada). Metformin hydrochloride tablet cores (500 mg/core) and Nifedipine double-layer tablet cores (60 mg/core) were gifts from National Pharmaceutical Engineering Research Center (Shanghai, China). Ammonium formate, sodium lauryl sulfate, methanol, and sodium hydrogen phosphate were purchased from National Pharmaceutical Chemical Reagent Co., Ltd. (Beijing, China). Acetonitrile was purchased from CINC High Purity Solvents Co., Ltd. (Shanghai, China). Formic acid was purchased from Shanghai Aladdin Bio-Chem Technology Co., Ltd. (Shanghai, China).

\section{Preparation Method for Thermoplastic-Coated Tablets} HHL-METF and HHL-NIF were prepared by the HHL method (refer to the preceding report ${ }^{1}$ ), respectively. The average content of METF tablet cores was $435.73 \pm 15.69 \mathrm{mg}$ per tablet core and the content of NIF double-layer tablet cores was $60 \mathrm{mg}$. For HHL-METF, abbreviated as M15-80, the membrane composition was cellulose acetate (CA): triethyl citrate (TEC): polyethylene glycol $1500($ PEG1500)=60: 25: 15 and the membrane thickness was $80 \mu \mathrm{m}$. For HHL-NIF, abbreviated as N5-90, the membrane composition was CA: TEC: PEG1500 = 70: 25: 5 and the membrane thickness was $90 \mu \mathrm{m}$. An orifice was drilled at the center of both sides of HHL-METF, while an orifice was only drilled at the center on the drug-containing side of HHL-NIF.

\section{Determination of Release Profiles and Drug-Release Models of the Thermoplastic-Coated Tablets}

The in vitro drug-release profiles of M15-80 and N5-90 were studied according to "Determination of Release Profiles" described in the previous article "New Technology of Thermoplastic Coating for Osmotic Pump Tablets: Study on in vitro Drug Release."3

The release data of M15-80 and N5-90 were fitted with drug-release models, such as zero-order, first-order, and the
Higuchi square-root of time release equations by calculating the correlation coefficient $r^{2},{ }^{4}$ respectively.

\section{Pharmacokinetic Study of HHL-METF}

\section{Chromatographic Condition}

High-performance liquid chromatography (HPLC, LC-20AT SPD-20A, Shimadzu Corporation, Japan) was used. The chromatographic conditions were decided by referring to relevant literature. ${ }^{5,6}$ The chromatographic column was XBridge $\mathrm{C}_{18}$ column $(4.6 \mathrm{~mm} \times 250 \mathrm{~mm}, 5 \mu \mathrm{m})$. The mobile phase was acetonitrile: $10 \mathrm{mmol} / \mathrm{L}$ sodium dihydrogen phosphate buffer (containing $7.5 \mathrm{mmol}$ of sodium dodecyl sulfate; the $\mathrm{pH}$ value was 5.5$)=32: 68$. The flow rate was $1 \mathrm{~mL} / \mathrm{min}$ and the detection wavelength was $233 \mathrm{~nm}$. The injection volume was $20 \mu \mathrm{L}$ and the column temperature was $45^{\circ} \mathrm{C}$.

\section{Preparation of Series Standard Solutions}

The standard substance of METF was weighed accurately (analytical balance, MS105DU, Mettler-Toledo), then dissolved in water and diluted to a concentration of $1.0 \mathrm{mg} / \mathrm{mL}$, which was used as a stock solution. Precise amounts of the stock solution were pipetted and diluted with water to prepare a series of standard solutions with concentrations of $4.0,10.0,20.0,40.0,100.0,160.0$, and $200.0 \mu \mathrm{g} / \mathrm{mL}$, respectively.

\section{Plasma Sample Processing Method}

A plasma sample $(200 \mu \mathrm{L})$ melted at room temperature was accurately pipetted into a $1.5 \mathrm{~mL}$ stoppered centrifuge tube, and $400 \mu \mathrm{L}$ of acetonitrile was added into the tube to precipitate proteins. The sample was eddied for 5 minutes (vortex mixer, XW-80C, Shanghai Medical University Instrument Factory) and then centrifuged for 15 minutes under the speed of $6641.0 \times g$ at room temperature (high-speed bench centrifuge, TGL-16B, Shanghai Anting Scientific Instrument Factory). Then the supernatant was transferred into a sample tube and stored at $4^{\circ} \mathrm{C}$ against exposure to light.

\section{Specificity and Standard Curve}

For specificity, the samples of blank plasma, blank plasma added standard solution, and plasma sample of Beagle dogs after drug administration were processed according to "Plasma Sample Processing Method" and then injected directly into the HPLC system, respectively. The retention time and separation degree of METF in plasma were investigated.

For the standard curve, the blank plasma was added the appropriate amount of METF standard solutions and the standard curve plasma samples with a series of concentrations of $0.20,0.50,1.00,2.00,5.00,8.00$, and $10.00 \mu \mathrm{g} / \mathrm{mL}$ were prepared, which were processed according to "Plasma Sample Processing Method."

\section{Experiment Design}

Six healthy adult Beagle dogs weighing $16.4 \pm 2.5 \mathrm{~kg}$ (three males and three females) were purchased from Shanghai Jiagan Biotechnology Co., Ltd. (SCXK [Shanghai] 2015-0005). 
Table 1 Mass spectrometric parameters

\begin{tabular}{|l|l|l|l|l|l|l|l|}
\hline Name & Note & Q1 $(\mathrm{m} / \mathrm{z})$ & Q3 $(\mathrm{m} / \mathrm{z})$ & $\begin{array}{l}\text { Dwell time } \\
(\mathrm{ms})\end{array}$ & $\begin{array}{l}\text { Q1 PreBias } \\
(\mathrm{V})\end{array}$ & $\begin{array}{l}\text { Collision Energy } \\
(\mathrm{V})\end{array}$ & $\begin{array}{l}\text { Q3 PreBias } \\
(\mathrm{V})\end{array}$ \\
\hline Nifedipine & Target & 347 & 315.15 & 100 & -13 & -10 & -21 \\
\hline Nifedipine-d6 & Internal standard (IS) & 353.1 & 318.15 & 100 & -13 & -10 & -21 \\
\hline
\end{tabular}

The experimental program was reviewed and approved by the Ethics Committee of the School of Pharmacy of Fudan University. A single cycle and single dose design with oral administration after meal was adopted. The Beagle dogs were fasted for 12 hours before meal, but they could drink water freely. M15-80 was placed at the base of the dog's tongue. After given $100 \mathrm{~mL}$ of water quickly, its jaws were closed immediately to ensure that the dog swallowed the tablet by itself. Then the Beagle dogs could drink water freely after administration and were fed 6 hours after administration.

Time points for blood collection were before administration (0 hour) and 0.5, 1, 2, 3, 4, 5, 6, 7, 8, 10, 12, 14, and 24 hours after administration. Two milliliters of blood was collected from the canine radial vein of forelimb, placed into a heparin-containing centrifuge tube, and centrifuged for 3 minutes under the speed of $1307.8 \times \mathrm{g}$ at room temperature (low-speed bench centrifuge, TDL-80-2B, Shanghai Anting Scientific Instrument Factory). Plasma in the upper layer was transferred into a $1.5 \mathrm{~mL}$ stoppered plastic tube and stored at $20^{\circ} \mathrm{C}$.

\section{Pharmacokinetic Study of HHL-NIF}

Chromatographic Condition

The liquid chromatography/triple quadrupole mass spectrometry (LC/MS, LCMS-8060, LabSolutions LCMS 5.82 SP1+ CLASS-Agent, Shimadzu Corporation, Japan) was used. The chromatographic conditions were decided by referring to relevant literature. ${ }^{7,8}$ The mobile phase was $60 \%$ acetonitrile (containing $5 \mathrm{mmol} / \mathrm{L}$ ammonium formate and $0.1 \%$ formic acid). The flow rate was $0.4 \mathrm{~mL} / \mathrm{min}$. The chromatographic column was Waters Acquity UPLC High Strength Silica (HSS) T3 $(2.1 \mathrm{~mm} \times 100 \mathrm{~mm}, 1.8 \mu \mathrm{m})$ and the guard column was Vanguard Bridged Ethylene Hybrid $(\mathrm{BEH}) \mathrm{C}_{18}(1.7 \mu \mathrm{m})$. The running time was 2 minutes. The column oven temperature was $35^{\circ} \mathrm{C}$ and the injection volume was $4 \mu \mathrm{L}$. Except the sample at 0.3 to 1 minute deserted as waste liquid, the rest sample during the running time was tested by MS through switching the postcolumn valve. The sample chamber temperature was $4^{\circ} \mathrm{C}$.

\section{Mass Spectrometry Conditions}

The mass spectrometer was run in positive ion multiple reaction monitoring (MRM) mode and its ionization mode was $\mathrm{ESI}^{+}$. The interface voltage was selected as $4 \mathrm{kV}$. The atomized gas flow rate and the heating gas flow rate were set at 3 and $10 \mathrm{~L} / \mathrm{min}$, separately. The interface temperature was $300^{\circ} \mathrm{C}$, the DL temperature was $250^{\circ} \mathrm{C}$, and the heating block temperature was $400^{\circ} \mathrm{C}$. The dry gas flow rate was $10 \mathrm{~L} / \mathrm{min}$.
Other mass spectrometric parameters of the compounds are shown in - Table 1.

\section{Solution Preparation and Biological Sample Pretreatment (Operated in Dark Place)}

Preparation of NIF Standard Curve Working Solutions About $10 \mathrm{mg}$ of the NIF standard substance was accurately weighed (analytical balance, MS105DU, Mettler-Toledo Company), placed in a $100 \mathrm{~mL}$ brown volumetric flask, dissolved and diluted to the mark with methanol. Then the solution was diluted with $50 \%$ methanol stepwise to prepare standard curve working solutions with concentrations of 500.0, 250.0, $100.0,50.0,25.0,5.0$, and $2.0 \mathrm{ng} / \mathrm{mL}$, respectively.

\section{Preparation of NIF Quality Control Sample Working Solutions}

About $10 \mathrm{mg}$ of NIF standard substance was accurately weighed, placed in a $100 \mathrm{~mL}$ brown volumetric flask, dissolved and diluted to the mark with methanol. Then the solution was diluted with $50 \%$ methanol stepwise to prepare quality control $(\mathrm{QC})$ working solutions with concentrations of $400.0,40.0,4.0$, and $2.0 \mathrm{ng} / \mathrm{mL}$, respectively.

\section{Internal Standard Working Solution}

An appropriate amount of NIF-d6 was weighed, placed in a $50 \mathrm{~mL}$ brown volumetric flask, dissolved with a small amount of methanol, and diluted to the mark with $50 \%$ methanol to obtain an internal standard working solution with a concentration of $40.0 \mathrm{ng} / \mathrm{mL}$.

\section{Standard Solution}

Each $1 \mathrm{~mL}$ of the $\mathrm{QC}$ working solution and the internal standard working solution with the concentration of 40.0 $\mathrm{ng} / \mathrm{mL}$ was pipetted into the same $50 \mathrm{~mL}$ brown volumetric flask and diluted to the mark with $50 \%$ methanol to obtain the standard solution of NIF and NIF-d6 with the concentration of $0.80 \mathrm{ng} / \mathrm{mL}$.

\section{Plasma Sample Processing Method}

A plasma sample $(100 \mu \mathrm{L})$ melted at room temperature was accurately pipetted into a $1.5 \mathrm{~mL}$ brown plastic tube. Each $10 \mu \mathrm{L}$ of $50 \%$ methanol and the internal standard working solution was successively added into the tube and mixed by shaking. Then $400 \mu \mathrm{L}$ of acetonitrile was added into the tube to precipitate protein. After eddied for 5 minutes, the sample was centrifuged for 15 minutes under the speed of 9 $563.0 \times g$ at room temperature (high-speed bench centrifuge, TGL-16B, Shanghai Anting Scientific Instrument Factory). Then the supernatant was transferred into a new sample 
tube and stored at $4^{\circ} \mathrm{C}$ against exposure to light. The sample should be determined within 43.5 hours.

\section{Specificity and Standard Curve}

For specificity, the samples of blank plasma, blank plasma added standard solution, the lowest limit of quantification (LLOQ $=0.20 \mathrm{ng} / \mathrm{mL}$ ) solution of the standard curve plasma sample, and plasma sample of Beagle dogs after drug administration were processed according to "Plasma Sample Processing Method" and then injected directly into the LC/MS, respectively. The retention time, separation degrees of NIF, and the interference from endogenous substances were investigated.

For the standard curve, plasma samples are available immediately before use. First, $90 \mu \mathrm{L}$ of blank plasma was pipetted into a $1.5 \mathrm{~mL}$ brown plastic tube and $10 \mu \mathrm{L}$ of the above series of NIF standard curve working solutions was precisely added to obtain NIF standard curve plasma samples with final concentrations of 50.00, 25.00, 10.00, 5.00, 2.50, 0.50 , and $0.20 \mathrm{ng} / \mathrm{mL}$, respectively. Then the samples were processed according to "Plasma Sample Processing Method" before determination. Each sample was prepared in duplicate.

\section{Experiment Design}

Fourteen days after the pharmacokinetic study of M15-80, the same six Beagle dogs were used in the pharmacokinetic study of N5-90. The Beagle dogs were fasted for 12 hours before dosing, but they could drink water freely. N5-90 was administered orally as M15-80, which was placed at the base of the dog's tongue. After given $90 \mathrm{~mL}$ of water, its jaws were closed immediately to ensure that the dog swallowed the tablet by itself. The Beagle dogs could drink water freely after administration and were fed 6 hours after administration.

Time points for blood collection were before administration (0 hour) and 1, 2, 3, 4, 5, 6, 7, 8, 10, 12, 24, and 36 hours after administration. Blood sample $(1.5 \mathrm{~mL})$ was collected from the canine radial vein of forelimb, placed into a heparincontaining centrifuge tube and centrifuged for 3 minutes under the speed of $1307.8 \times g$ at room temperature. Plasma in the upper layer was transferred into a $1.5 \mathrm{~mL}$ stoppered plastic tube and stored at $20^{\circ} \mathrm{C}$.

\section{Determination of Blood Concentration and Pharmacokinetic Parameter Calculation}

The concentrations of METF and NIF in plasma samples of Beagle dogs at different time points were determined by the established HPLC method and LC/MS method, respectively. The pharmacokinetic parameters of M15-80 and N5-90 were calculated by DAS 2.1.1 software, including $\mathrm{AUC}_{0-t}$, $\mathrm{AUC}_{0-\infty}, c_{\max }, t_{\max }, t_{1 / 2}$, and MRT. The area under the curve of drug concentration-time $\left(\mathrm{AUC}_{0-t}\right)$ was calculated by the trapezoidal method, and $t_{\max }$ and $c_{\max }$ were represented by measured values.

\section{In vitro-in vivo Correlation Analysis}

The in vivo release profiles of M15-80 and N5-90 were fitted with pharmacokinetic compartment models using DAS 2.1.1

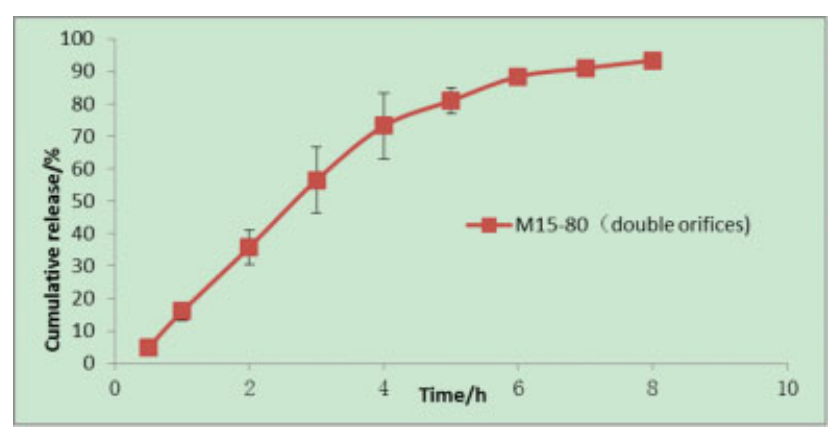

Fig. 1 Release profile of M15-80 $(n=6)$.

software, respectively. And the in vitro-in vivo correlation was analyzed according to the "in vitro-in vivo correlation" section in the guideline for sustained release, controlled release, and delayed release preparations (9013) of Chinese Pharmacopoeia (volume IV, 2015 edition). ${ }^{4}$

\section{Results and Discussion}

\section{Release Profiles and Drug-Release Models}

The release profiles of M15-80 and N5-90 are shown in -Figs. 1 and 2. ${ }^{3}$ The results showed that both tablets released slowly in vitro.

The release rates of M15-80 and N5-90 are shown in -Figs. 3 and 4. The release rate of M15-80 was constant at $89.96 \pm 10.67 \mathrm{mg} / \mathrm{h}$ from 1 to 4 hours, and the cumulative release of METF at 4 hours was $73.17 \%$. Also, the release rate of N5-90 was constant at $6.71 \pm 0.82 \mathrm{mg} / \mathrm{h}$ from 2 to 8 hours, and the cumulative release of NIF at 8 hours was $80.23 \%$. These results indicated that the release rates of both tablets were zero-order at certain time.

The release data of M15-80 and N5-90 were fitted with drug-release models, such as zero-order, first-order, and the Higuchi square-root of time release equations by calculating the correlation coefficient $r^{2}$, respectively. The results are shown in -Table 2. The results indicated that M15-80 displayed approximately zero-order release characteristics within 6 hours and N5-90 displayed approximately zeroorder release characteristics within 12 hours.

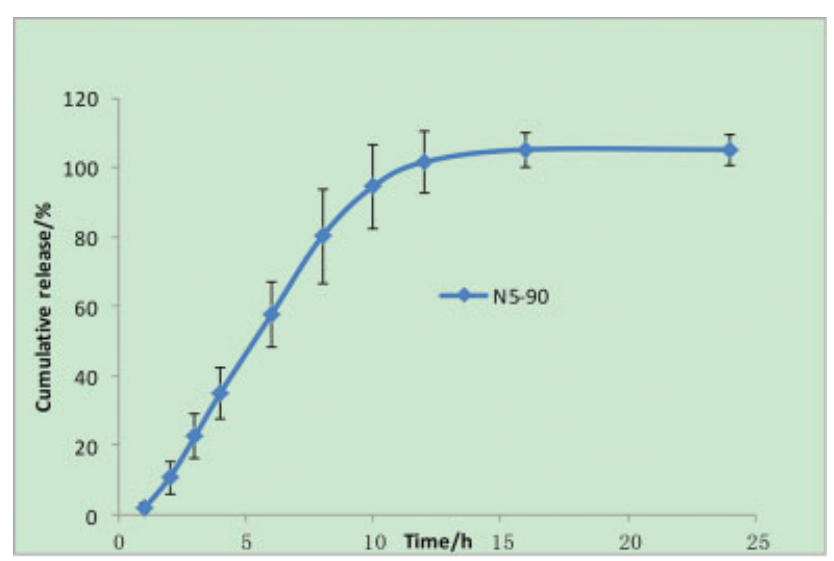

Fig. 2 Release profile of $\mathrm{N} 5-90(n=6)$. 


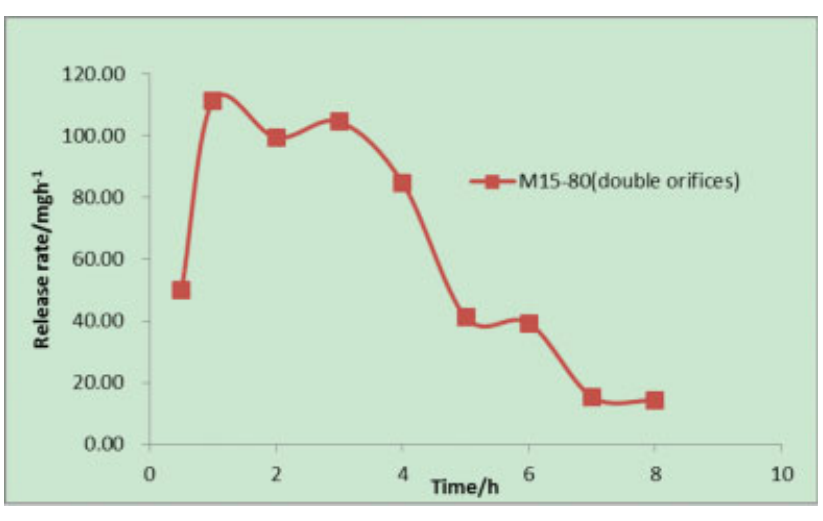

Fig. 3 Release rate-time profile of M15-80.

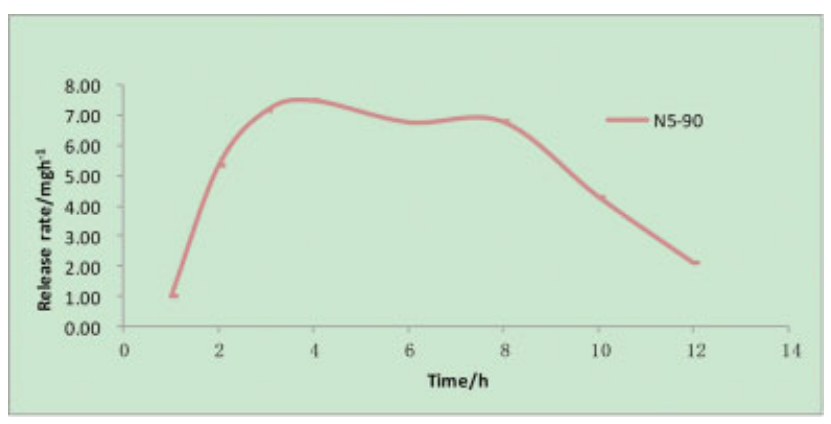

Fig. 4 Release rate-time profile of N5-90.

\section{Pharmacokinetic Study of HHL-METF}

\section{Specificity}

The chromatogram results of METF specificity are shown in - Fig. 5. It was shown that the retention time of METF was 14.1 minutes under this chromatographic condition, and no other chromatographic peak interferences were observed around 14.1 minutes. The separation degree of METF was good, and the endogenous substance did not affect the determination of the METF.

\section{Standard Curve}

The linear test results of the plasma concentration of METF are shown in - Table 3. The standard curve was calculated by the weighted least-square method $\left(1 / c^{2}\right)$. The regression equation was $Y=47647.99 c-14262.96(r=0.9958)$, where $Y$ is the sample peak area and $c$ is the concentration of series standard solutions. The results showed that METF had a good linear relationship within the concentration range of 0.20 to $10.00 \mu \mathrm{g} / \mathrm{mL}$, and the LLOQ in plasma was $0.20 \mu \mathrm{g} / \mathrm{mL}$.

Blood Concentration and Pharmacokinetic Parameters The mean blood concentration-time curve of the six Beagle dogs after single-dose oral administration of M15-80 is shown in -Fig. 6. The results showed that the METF of M15-80 had the characteristics of slow absorption in vivo. The concentration-time curve of conventional METF-OPTs (EOP) in fasting Beagle dogs after oral administration reported in the literature ${ }^{5}$ is shown in -Fig. 7 (red line), which was similar to the mean blood concentration-time curve of M15-80 in our study.

The dosage of M15-80 in our pharmacokinetic study was different from that of the EOP tablets reported in the literature. ${ }^{5}$ Based on the linear pharmacokinetic characteristics of METF, ${ }^{9}$ the parameters of M15-80 were multiplied by the correction factor of $1.71(45.45 / 26.57=1.71)$ before comparing with that of EOP. The results in - Table 4 showed that: (1) the difference in bioavailability $(F)$ between M15-80 and EOP was little, and the average relative bioavailability $\mathrm{F}_{0-t}$ of $\mathrm{M} 15-80$ relative to EOP was $77.98 \%$. (2) The $c_{\max }$ ratio of M15-80 relative to EOP was $103.14 \%$. (3) The corrected curve of M15-80 was basically consistent with EOP, as shown in - Fig. 7 (blue line), suggesting that M15-80 prepared by thermoplastic coating had the same function of controlling drug release as the conventional OPTs. In addition, M15-80 was administered to dogs after meal in our study, while EOP was administered under the fasting state in the literature, indicating that the release profile of M15-80 in vivo was not affected by food, and M15-80 possessed the drugrelease characteristics of OPTs.

\section{In vitro-in vivo Correlation}

The pharmacokinetic model of M15-80 in Beagle dogs was analyzed using DAS 2.1.1 software. The fitting result was a two-compartment model. When the weight factor was $1 / c$, the comprehensive fitting effect was optimal, and the pharmacokinetic parameters were as follows: $k_{10}=2.365$, $k_{12}=0.017, k_{21}=0.275, \beta=0.198$. The absorption rates $(f)$

Table 2 Correlation coefficient $r^{2}$ of various drug-release models fitted by M15-80 and N5-90

\begin{tabular}{|l|l|l|l|l|}
\hline Tablets & Time $(\mathbf{h})$ & Zero-order & First-order & Higuchi's model \\
\hline \multirow{5}{*}{ M15-80 } & 16 & 0.670 & 0.841 & 0.838 \\
\cline { 2 - 5 } & 12 & 0.765 & 0.920 & 0.903 \\
\cline { 2 - 5 } & 8 & 0.903 & 0.993 & 0.973 \\
\cline { 2 - 5 } & 6 & 0.959 & 0.990 & 0.990 \\
\hline N5-90 & 24 & 0.788 & 0.871 & 0.913 \\
\cline { 2 - 5 } & 12 & 0.988 & 0.991 & 0.988 \\
\cline { 2 - 5 } & 6 & 0.993 & 0.986 & 0.965 \\
\hline
\end{tabular}




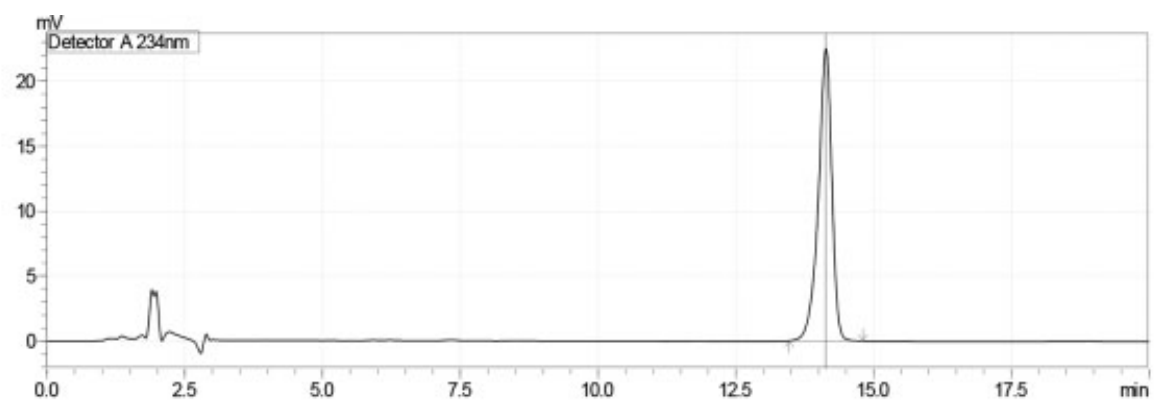

A

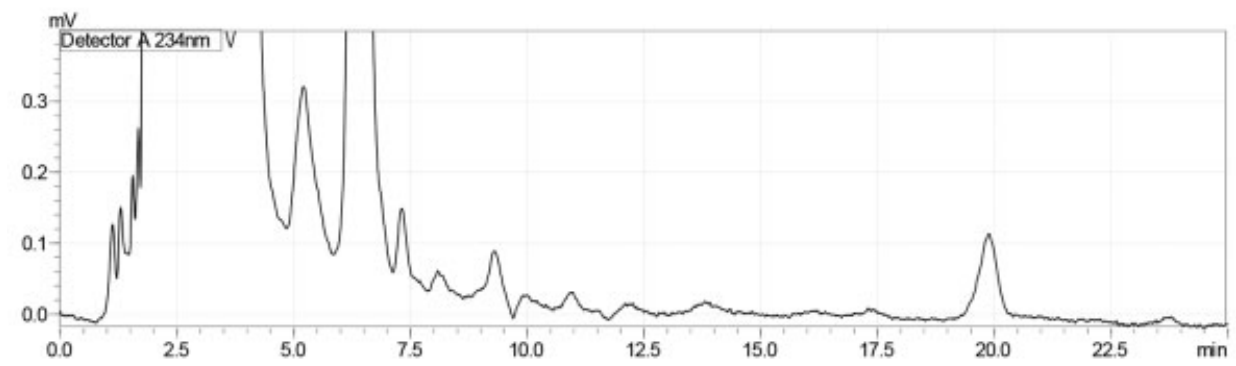

B
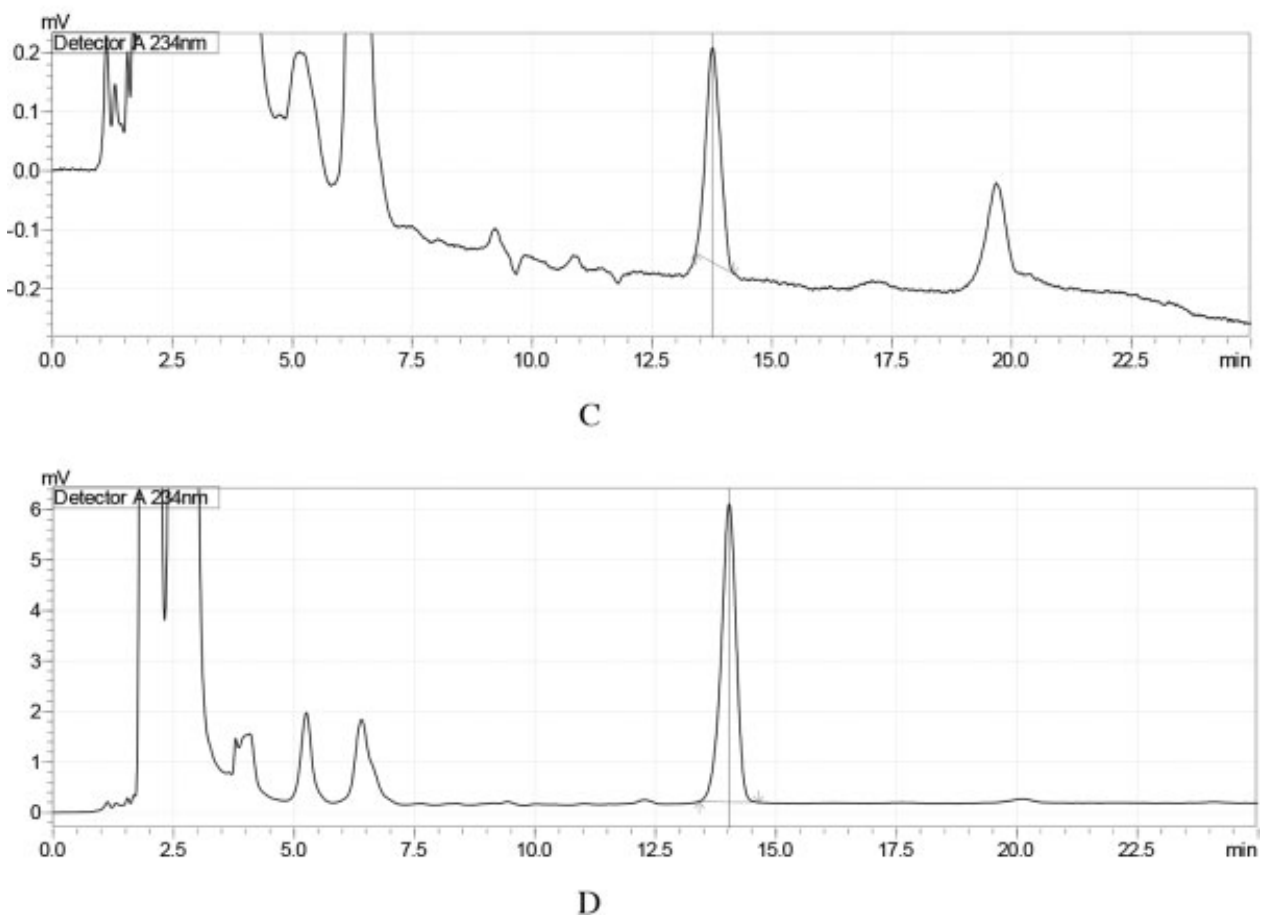

Fig. 5 The chromatograms of METF specificity. (A) METF standard solution $(4.0 \mu \mathrm{g} / \mathrm{mL})$; (B) blank plasma sample from No.1 Beagle dogs; (C) blank plasma added METF standard solution (LLOQ $=0.20 \mu \mathrm{g} / \mathrm{mL}$ ); and (D) the 5-hour plasma sample from No.1 Beagle dogs after taking M15-80 orally. LLOQ, lower limit of quantitation.

of the drug at different times in vivo were calculated according to the Loo-Riegelman equation. ${ }^{4}$

$$
f=\frac{c_{t}+k_{10} \int_{0}^{t} c \mathrm{dt}+\frac{\left(\mathrm{X}_{\mathrm{P}}\right)_{t}}{V_{c}}}{k_{10} \int_{0}^{\infty} c \mathrm{dt}}
$$

Where $c_{t}$ is the plasma concentration at time $t,\left(\mathrm{X}_{\mathrm{p}}\right)_{\mathrm{t}}$ is the amount of drug in the peripheral chamber, and $V_{c}$ is the apparent distribution volume in the central chamber. By inputting the corresponding parameters into the 3P97 pharmacokinetic program software, the in vivo absorption rate was calculated. The results are shown in - Table 5 .

The linear equation $f=0.7375 R-15.943(r=0.9591)$ was obtained by regression analysis of the in vitro release rate and in vivo absorption rate. The corresponding time points were 6 (excluding 0 hour), the statistical degree of freedom $(v)$ was 5 , and the calculated in vitro-in vivo correlation coefficient $(r=0.9591)$ was larger than the 
Table 3 Linear test results of plasma concentration of METF

\begin{tabular}{|l|l|l|}
\hline $\begin{array}{l}\text { Standard } \\
\text { concentration } \\
(\mu \mathrm{g} / \mathrm{mL})\end{array}$ & $\begin{array}{l}\text { Concentration } \\
(\mu \mathrm{g} / \mathrm{mL})\end{array}$ & Accuracy (\%) \\
\hline 0.20 & 0.192 & 95.7 \\
\hline 0.50 & 0.532 & 106.2 \\
\hline 1.00 & 1.087 & 108.5 \\
\hline 2.00 & 2.082 & 103.9 \\
\hline 5.00 & 5.246 & 104.7 \\
\hline 8.00 & 6.993 & 87.2 \\
\hline 10.00 & 9.397 & 93.8 \\
\hline
\end{tabular}

Table 4 Comparison of main pharmacokinetic parameters between M15-80 and OPTs reported in the literature

\begin{tabular}{|l|l|l|l|}
\hline Parameters & M15-80 & $\begin{array}{l}\text { M15-80 } \times 1.71 \\
\text { (correction) }\end{array}$ & $\begin{array}{l}\text { EOP } \\
\text { tablet }^{5}\end{array}$ \\
\hline Dosage $(\mathrm{mg} / \mathrm{kg})$ & 26.57 & 45.45 & 45.45 \\
\hline$C_{\max }(\mathrm{mg} / \mathrm{L})$ & 3.84 & 6.56 & 6.36 \\
\hline$t_{\max }(\mathrm{h})$ & 4.83 & 4.83 & 4.08 \\
\hline $\mathrm{AUC}_{0-t}(\mathrm{mg} / \mathrm{L} \cdot \mathrm{h})$ & 24.00 & 41.05 & 52.64 \\
\hline $\mathrm{AUC}_{0-\infty}(\mathrm{mg} / \mathrm{L} \cdot \mathrm{h})$ & 24.54 & 41.97 & 56.43 \\
\hline$t_{1 / 2}(\mathrm{~h})$ & 4.12 & - & - \\
\hline
\end{tabular}

Table 5 The in vivo and in vitro cumulative percentages of M15-80

\begin{tabular}{|l|l|l|l|l|l|l|}
\hline Time $(\mathrm{h})$ & $\mathbf{1}$ & $\mathbf{2}$ & $\mathbf{3}$ & $\mathbf{4}$ & $\mathbf{5}$ & $\mathbf{6}$ \\
\hline In vitro release rate $(R / \%)$ & 16.08 & 35.86 & 56.55 & 73.17 & 80.79 & 88.41 \\
\hline In vivo absorption rate $(f / \%)$ & 2.03 & 7.66 & 18.84 & 32.23 & 45.70 & 56.64 \\
\hline
\end{tabular}

critical correlation coefficient $(r=0.951)$ when the $v$ value was 5 and the statistical significance $(P)$ was 0.001 . This indicated that there was a good correlation between in vitro release and in vivo absorption of M15-80 prepared by

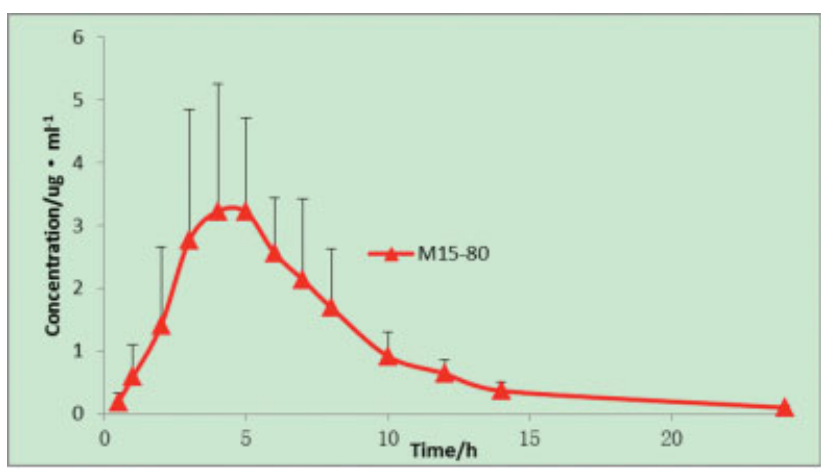

Fig. 6 Mean plasma concentration-time curve of the M15-80 in fed Beagle dogs after oral administration $(n=6)$.

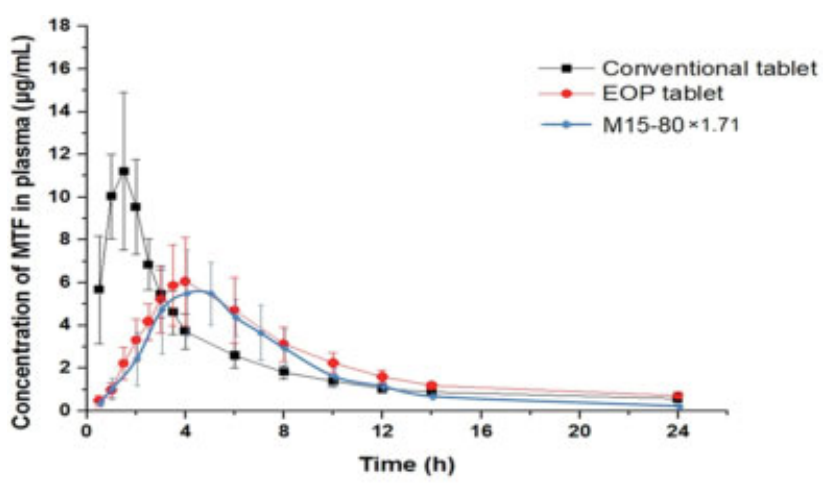

Fig. 7 Mean plasma concentration-time curves of conventional METF tablet and METF EOP tablet in fasting Beagle dogs ${ }^{5}$ and the corrected mean plasma concentration-time curve of M15-80 in fed Beagle dogs after oral administration $(n=6)$. thermoplastic coating, therefore the in vivo drug absorption could be predicted by in vitro drug release. ${ }^{10,11}$

\section{Pharmacokinetic Study of HHL-NIF}

\section{Specificity}

The chromatographic peak of NIF and the corresponding internal standard peak were qualitatively determined by chromatographic retention time and ion pair, and the chromatograms of specificity in - Fig. 8 show that the retention time of NIF was approximately 1.57 minutes and the internal standard NIF-d6 was approximately 1.54 minutes. Endogenous substances did not interfere with the main peak and internal standard peak.

\section{Standard Curve}

The linear test results of plasma concentration of NIF are shown in - Table 6 . The standard curve was calculated by using the weighted least-square method $(1 / c)$. The regression equation was $Y=0.298474 c-6.0416 \times 10^{-3}(r=0.9999)$, where $Y$ is the ratio of the NIF peak area to the internal standard peak area and $c$ is the concentration of series standard solutions. The results showed that NIF had a good linear relationship within the concentration range of 0.20 to $50.00 \mathrm{ng} / \mathrm{mL}$, and the LLOQ in plasma was $0.20 \mathrm{ng} / \mathrm{mL}$.

\section{Blood Concentration and Pharmacokinetic Parameters}

The mean blood concentration-time curve of the six Beagle dogs after single-dose oral administration of N5-90 is shown in - Fig. 9. It shows that the plasma concentration of NIF was above $5 \mathrm{ng} / \mathrm{mL}$ in the range of 2 to 20 hours, indicating that N5-90 released slowly in the gastrointestinal tract of dogs and maintained a relatively stable blood concentration, which was consistent with the phenomenon reported in the literature that NIF was absorbed in the whole intestine $^{12,13}$ and was suitable for development as a controlled 


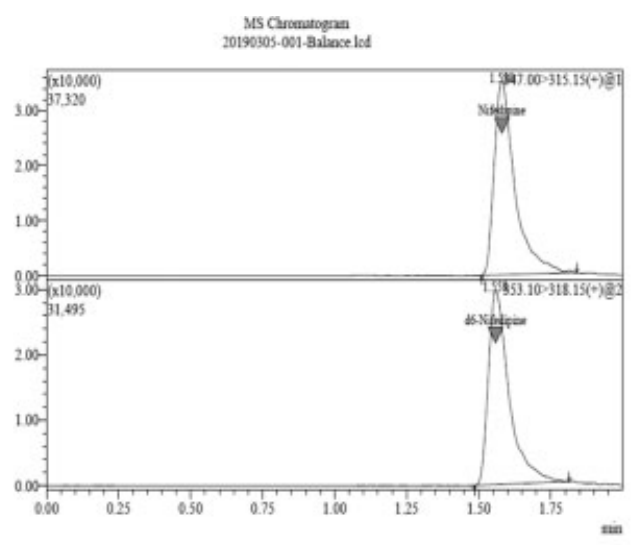

A

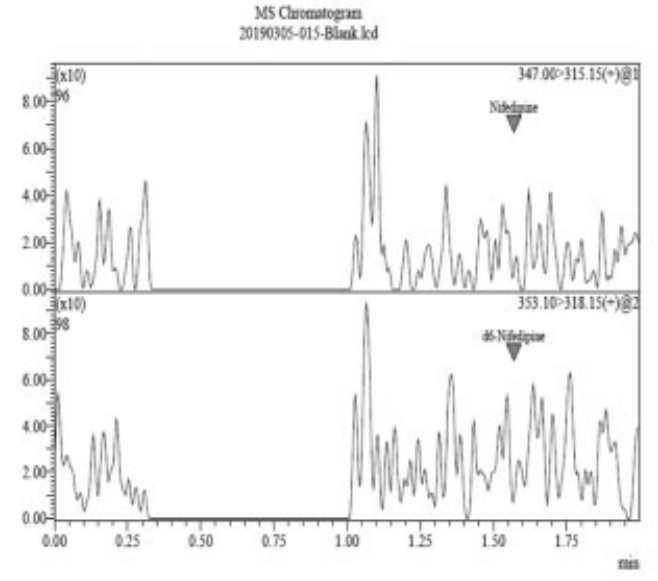

C

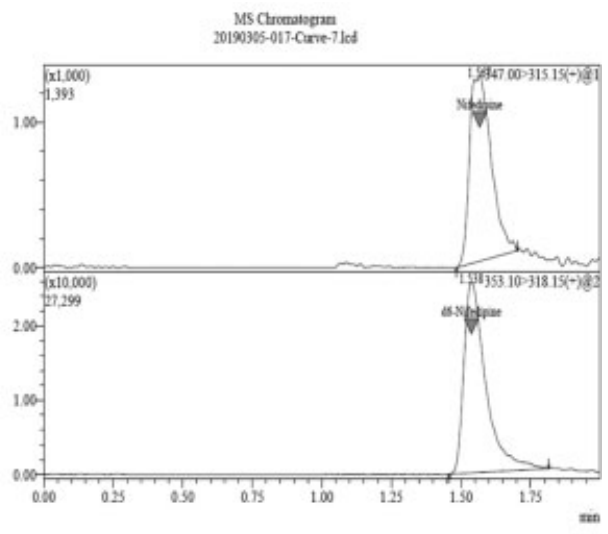

B

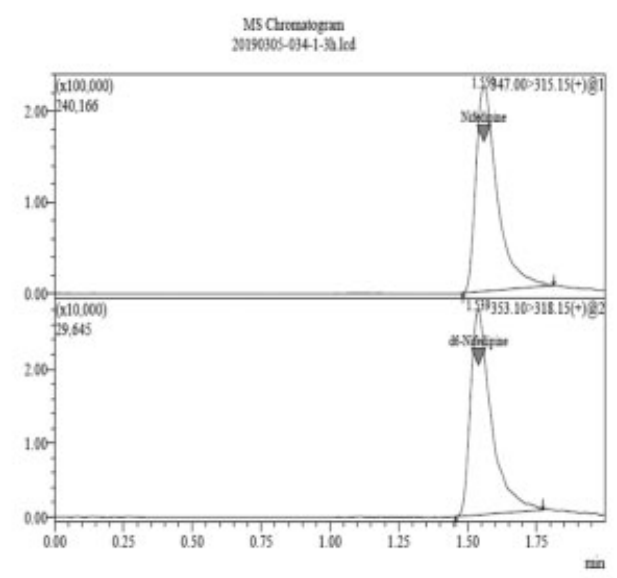

D

Fig. 8 The chromatograms of NIF specificity. (A) Standard solution of NIF and NIF-d6; (B) lower limit of quantitation (LLOQ =0.20 ng/mL); (C) blank plasma sample; (D) the 3-hour plasma sample from No.1 Beagle dogs after taking N5-90 orally.

release preparation. ${ }^{12}$ According to Grundy et al's research, ${ }^{14}$ the double-peak phenomenon in the blood concentrationtime curve of N5-90 was not caused by the enterohepatic circulation of NIF, but by the slow release of N5-90 in the whole intestinal tract of dogs, which was consistent with the observation of the double-peak phenomenon of NIF in the presence of grapefruit juice that could delay gastric emptying. ${ }^{14}$
The main pharmacokinetic parameters of N5-90 after a single oral dose in fasting Beagle dogs are shown in - Table 7. The $t_{1 / 2}$ was $6.55 \pm 2.94 \mathrm{~h}$, which was longer than that of the common NIF preparation reported in the literature $\left(t_{1 / 2}=2\right.$ hours), ${ }^{15}$ indicating that N5-90 possessed the characteristics of sustained release preparation in vivo.

Pan et al studied the pharmacokinetics of NIF dualchamber osmotic pump controlled release tablets (Adalat

Table 6 Linear test results of plasma concentration of NIF

\begin{tabular}{|l|l|l|l|l|}
\hline $\begin{array}{l}\text { Standard concentration } \\
(\mathbf{n g} / \mathrm{mL})\end{array}$ & $\begin{array}{l}\text { Concentration 1 } \\
(\mathbf{n g} / \mathrm{mL})\end{array}$ & $\begin{array}{l}\text { Accuracy } \\
(\%)\end{array}$ & $\begin{array}{l}\text { Concentration 2 } \\
(\mathbf{n g} / \mathrm{mL})\end{array}$ & $\begin{array}{l}\text { Accuracy } \\
(\%)\end{array}$ \\
\hline 0.20 & 0.185 & 92.5 & 0.201 & 100.5 \\
\hline 0.50 & 0.524 & 104.8 & 0.506 & 101.2 \\
\hline 2.50 & 2.456 & 98.2 & 2.491 & 99.6 \\
\hline 5.00 & 5.237 & 104.7 & 4.937 & 98.7 \\
\hline 10.00 & 9.840 & 98.4 & 9.890 & 98.9 \\
\hline 25.00 & 25.350 & 101.4 & 25.620 & 102.5 \\
\hline 50.00 & 50.090 & 100.2 & 49.070 & 98.1 \\
\hline
\end{tabular}




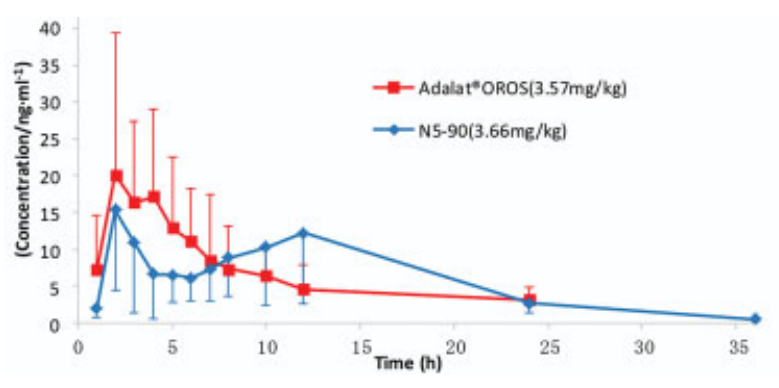

Fig. 9 Comparison of mean plasma concentration-time curves of N5-90 and NIF OPTs reported in the literature ${ }^{7}$ in fasting Beagle dogs after oral administration $(n=6)$. OPTs, osmotic pump tablets.
Table 7 Comparison of main pharmacokinetic parameters between N5-90 and OPTs reported in the literature

\begin{tabular}{|c|c|c|}
\hline Parameter & N5-90 & Adalat $\mathrm{OROS}^{7}$ \\
\hline$A \cup C_{0-t}(\mu \mathrm{g} / \mathrm{L} \cdot \mathrm{h})$ & $209.50 \pm 132.00$ & $152.80 \pm 59.40$ \\
\hline $\mathrm{AUC}_{0-\infty}(\mu \mathrm{g} / \mathrm{L} \cdot \mathrm{h})$ & $215.00 \pm 133.40$ & $173.47 \pm 75.99$ \\
\hline$t_{1 / 2}(\mathrm{~h})$ & $6.55 \pm 2.94$ & $5.30 \pm 3.83$ \\
\hline$t_{\max }(\mathrm{h})$ & $7.17 \pm 5.31$ & $4.00 \pm 1.90$ \\
\hline$c_{\max }(\mu \mathrm{g} / \mathrm{L})$ & $17.44 \pm 11.63$ & $30.30 \pm 16.60$ \\
\hline $\mathrm{MRT}_{0-t}(\mathrm{~h})$ & $12.20 \pm 3.21$ & - \\
\hline $\mathrm{MRT}_{0-\infty}$ (h) & $13.23 \pm 4.20$ & - \\
\hline
\end{tabular}

Table 8 The in vitro and in vivo cumulated percentages of N5-90

\begin{tabular}{|l|l|l|l|l|l|l|l|l|}
\hline Time $(\mathrm{h})$ & $\mathbf{1}$ & $\mathbf{2}$ & $\mathbf{3}$ & $\mathbf{4}$ & $\mathbf{6}$ & $\mathbf{8}$ & $\mathbf{1 0}$ & $\mathbf{1 2}$ \\
\hline $\operatorname{In}$ vitro release rate $(R / \%)$ & 1.67 & 10.58 & 22.51 & 35.00 & 57.60 & 80.23 & 94.58 & 101.60 \\
\hline In vivo absorption rate $(f / \%)$ & 2.49 & 20.14 & 30.77 & 35.96 & 45.57 & 58.49 & 74.50 & 93.37 \\
\hline
\end{tabular}

OROS, Bayer) in Beagle dogs. ${ }^{7}$ The obtained mean concentration-time curve was compared with the results of our study, as shown in - Fig. 9: (1) according to the mean concentration-time curve, N5-90 displayed the characteristic of controlled release, and the in vivo release of N5-90 was slower. (2) According to the pharmacokinetic parameters, the average relative bioavailability $\mathrm{F}_{0-t}$ of $\mathrm{N} 5-90$ relative to Adalat OROS was $137.11 \%(n=6)$, and $\mathrm{F}_{0-\infty}$ was $120.75 \%(n=6)$. The $c_{\max }$ was $57.58 \%$ and the $t_{\max }$ was $179.3 \%$. (3) The duration time of plasma concentration above $5 \mathrm{ng} / \mathrm{mL}$ of $\mathrm{N} 5-90$ was longer than that of Adalat OROS. The above results further confirmed that N5-90 prepared by thermoplastic coating had the function of controlling drug release in vivo.

\section{In vitro-in vivo Correlation}

The pharmacokinetic model of N5-90 in Beagle dogs was analyzed using DAS 2.1.1 software. The fitting result was a two-compartment model, which was consistent with the pharmacokinetic study of the conventional OPTs reported in the literature. ${ }^{16}$ The pharmacokinetic parameters were as follows: $k_{10}=0.964, k_{12}=0.798, k_{21}=0.169, \beta=0.15$. The absorption rates $(f)$ of NIF at different times in vivo were calculated according to the Loo-Riegelman equation. The results are shown in - Table 8 .

The linear equation $f=0.742 R+7.7112(r=0.9726)$ was obtained by regression analysis of the in vitro release rate and in vivo absorption rate. The corresponding time points were 8 (excluding 0 hour), the statistical degree of freedom $(v)$ was 7 , and the obtained in vitro-in vivo correlation coefficient $(r=0.9726)$ was larger than the critical correlation coefficient $(r=0.898)$ when the $v$ value was 7 and the statistical significance $(P)$ was 0.001 . This indicated that there was a good correlation between the in vitro release and in vivo absorption of N5-90 prepared by thermoplastic coating, which was an important characteristic of OPTs.

\section{Conclusion}

Two kinds of thermoplastic-coated tablets (M15-80 and N5-90) were prepared via the new thermoplastic coating technology (HHL method). The in vitro release studies confirmed that both thermoplastic-coated tablets had the function of controlling drug release and the characteristics of zero-order release within a certain period of time. The blood concentration-time curve and main pharmacokinetic parameters of M15-80 in Beagle dogs showed a sustained release effect when compared with common METF tablets, and were similar to those of the conventional METF OPTs. What's more, the pharmacokinetic parameters of M15-80 were not affected by food. The blood concentration-time curve and main pharmacokinetic parameters of N5-90 in Beagle dogs were also similar to those of the conventional NIF OPTs. This suggested that the similarity between thermoplastic-coated tablets and conventional OPTs could be achieved by adjusting the membrane formulation and thickness. Thus, the two thermoplastic-coated tablets prepared via the HHL method have the effects of controlling drug release in vivo and a good in vitro-in vivo correlation, which means that the in vivo drug absorption could be effectively predicted by in vitro drug release.

The pharmacokinetic study reported in this article further demonstrated that the new thermoplastic coating technology could replace the conventional spray coating to manufacture OPTs, which laid a solid foundation for the industrialization and clinical application of thermoplastic coating. 


\section{Funding}

This research work was supported by National Pharmaceutical Engineering Research Center and R\&D Center of China Traditional Chinese Medicine Holding Co., Ltd.

\section{Conflict of Interest}

The authors declare no conflicts of interest.

\section{References}

1 Yuan CP, Hou HM, Qian MY, et al. A new thermoplastic coating for osmotic pump tablets I. Development of preparation process. Carol J Pharm 2019;50(01):48-58

2 Yuan CP, Hou HM, Ou SY, et al. A new thermoplastic coating for osmotic pump tablets II. Evaluation of the coating membrane. Carol J Pharm 2019;50(02):165-176

3 Yuan CP, Hou HM, Ou SY, et al. New technology of thermoplastic coating for osmotic pump tablets: study on in vitro drug release. Pharm Fronts 2019;1(01):e1-e10

4 Chinese Pharmacopoeia Commission. The Pharmacopoeia of the People's Republic of China 2015 ed. Vol. IV. Beijing: China Medical Science Press; 2015:368

5 Pan H, Jing H, Yang X, et al. Synchronized and controlled release of metformin hydrochloride/glipizide from elementary osmotic delivery. Drug Dev Ind Pharm 2017;43(05):780-788

6 Yin J, Feng Y, Lu J, et al. Preparation of novel metformin hydrochloride osmotic pump controlled release tablets and study on pharmacokinetics in Beagle dogs. Zhongguo Xin Yao Zazhi 2012; 21(20):2429-2433

7 Pan X, Zhou S, Fu Q et al. Determination of nifedipine in dog plasma by high-performance liquid chromatography with tandem mass spectrometric detection. Biomed Chromatogr 2014;28 (07):1036-1040
8 Zhang DL, Yuan CP, Ren XH, et al. Determination of nifedipine in human plasma by high performance liquid chromatographytandem mass spectrometry. Zhongguo Yiyuan Yaoxue Zazhi 2012;32(03):224-227

9 Choi YH, Kim SG, Lee MG. Dose-independent pharmacokinetics of metformin in rats: hepatic and gastrointestinal first-pass effects. J Pharm Sci 2006;95(11):2543-2552

10 Qin C, He W, Zhu C, et al. Controlled release of metformin hydrochloride and repaglinide from sandwiched osmotic pump tablet. Int J Pharm 2014;466(1-2):276-285

11 He W, Li Y, Zhang R, et al. Gastro-floating bilayer tablets for the sustained release of metformin and immediate release of pioglitazone: preparation and in vitro/in vivo evaluation. Int J Pharm 2014;476(1-2):223-231

12 Bode $\mathrm{H}$, Brendel $\mathrm{E}$, Ahr G, et al. Investigation of nifedipine absorption in different regions of the human gastrointestinal (GI) tract after simultaneous administration of 13C- and 12Cnifedipine. Eur J Clin Pharmacol 1996;50(03):195-201

13 He Q Yang J. Development of digital gastrointestinal model and its application in absorption prediction of nifedipine sustained-release tablets. J Chin Pharm Univ 2012;43(01): 85-91

14 Grundy JS, Eliot LA, Kulmatycki KM, et al. Grapefruit juice and orange juice effects on the bioavailability of nifedipine in the rat. Biopharm Drug Dispos 1998;19(03):175-183

15 Pollak PT, Herman RJ, Feldman RD. Therapeutic differences in 24-h ambulatory blood pressures in patients switched between bioequivalent nifedipine osmotic systems with differing delivery technologies. Clin Transl Sci 2017;10(03): 217-224

16 Yan G, Li H, Zhang R, et al. Preparation and evaluation of a sustained-release formulation of nifedipine HPMC tablets. Drug Dev Ind Pharm 2000;26(06):681-686 